\title{
"Like a Vibration Cascading through the Body": Energy-Like Somatic Experiences Reported by Western Buddhist Meditators
}

\author{
David J. Cooper ${ }^{1}$, Jared R. Lindahl ${ }^{2}$, Roman Palitsky ${ }^{1}$ (D) and Willoughby B. Britton ${ }^{1, *(D)}$ \\ 1 Department of Psychiatry and Human Behavior, Brown University, Providence, RI 02906, USA; \\ david_cooper_1@brown.edu (D.J.C.); roman_palitsky@brown.edu (R.P.) \\ 2 Department of Religious Studies, Brown University, Providence, RI 02912, USA; jared_lindahl@brown.edu \\ * Correspondence: willoughby_britton@brown.edu
}

check for updates

Citation: Cooper, David J., Jared R. Lindahl, Roman Palitsky, and Willoughby B. Britton. 2021. "Like a Vibration Cascading through the Body": Energy-Like Somatic Experiences Reported by Western Buddhist Meditators. Religions 12: 1042. https://doi.org/10.3390/ rel12121042

Academic Editor: Terje Sparby

Received: 10 October 2021

Accepted: 17 November 2021

Published: 24 November 2021

Publisher's Note: MDPI stays neutral with regard to jurisdictional claims in published maps and institutional affiliations.

Copyright: (c) 2021 by the authors. Licensee MDPI, Basel, Switzerland. This article is an open access article distributed under the terms and conditions of the Creative Commons Attribution (CC BY) license (https:// creativecommons.org/licenses/by/ $4.0 /)$.

\begin{abstract}
There are numerous historical and textual references to energy-like somatic experiences (ELSEs) from religious traditions, and even a few psychological studies that have documented related phenomena. However, ELSEs remain an understudied effect of meditation in contemporary research. Based upon narratives from a large qualitative sample of Buddhist meditators in the West reporting meditation-related challenges, this paper offers a unique glimpse into how ELSEs play out in the lives of contemporary meditation practitioners and meditation experts. Departing from studies presuming a "kundalini awakening" framework, this paper presents a broader scope for understanding ELSEs by describing the metaphors practitioners used when speaking about them; the trajectories and impacts of ELSEs, including the factors that were reported as influencing their nature or trajectory; the various ways in which they were interpreted and appraised by practitioners, teachers, and specialists, such as doctors and therapists; and how practitioners responded to them or managed them with particular remedies. Deciding how to interpret and manage ELSEs entailed recruiting frameworks from within and/or beyond the meditator's specific Buddhist lineage.
\end{abstract}

Keywords: energy-like somatic experiences; meditation-related challenges; kundalini

PACS: J0101

\section{Introduction}

\subsection{The Study of Meditation Practices and Meditation Experiences}

Research on the practice of meditation and the resultant experiences has been increasing in recent decades, in large part because of the clinical and commercial applications of practices such as mindfulness. Scientific research on long-term meditators from Buddhist traditions has investigated the effects of meditation on cognition, perception, and emotion, in particular (e.g., Brefczynski-Lewis et al. 2007; Lutz et al. 2008; MacLean et al. 2010), although research on other less investigated areas, such as the impacts of meditation on arousal and senses of self is also underway (e.g., Amihai and Kozhevnikov 2014; DorZiderman et al. 2013). Nevertheless, historians of Buddhist traditions are right to point out the methodological problems with this enterprise, as well as the limited conclusions that can be derived from employing scientific research on only a small range of practices and outcomes in comparison to the much broader scope that can be found across Buddhist traditions, both historically and in the present day (Lopez 2008; McMahan 2011; Sharf 2015; Waldron 2017). Furthermore, qualitative research on contemporary Buddhist meditation practitioners has shown that various Buddhist meditation practices can have effects and impacts on many aspects of human experience, and not all of these experiences are positive (Buttner 2016; Lindahl et al. 2017; Kaselionyte and Gumley 2017).

A range of unusual or anomalous somatic experiences have long been associated with meditation. Some Buddhist practices intentionally direct the flow of "subtle body energies" as a means of impacting physiology, mental states, and karma (Chaoul 2006; 
Kongtrul 2007). In other contexts, energy-like somatic experiences (ELSEs) are viewed as meditation-induced side effects requiring mitigation (Samuel 2019), or as the root cause of other difficulties, such as those associated with "meditation sickness" (Greene 2021; Hakuin 2009). Despite the extensive research on long-term meditators, reports of ELSEs and practices associated with them have received little scientific attention (Kozhevnikov et al. 2013).

This paper draws upon data from the Varieties of Contemplative Experience (VCE) project, a mixed-methods study of meditation-related challenges as reported by Buddhist meditation practitioners and meditation experts in the West. The study also includes information about how practitioners and experts interpreted and responded to meditationrelated challenges (Lindahl et al. 2017). From practitioner and expert narratives, the VCE study documented nearly sixty distinct meditation-related challenges across multiple domains of human experience. The most commonly reported challenge in the somatic domain was "somatic energies". The present paper offers a comprehensive analysis of what we will call "energy-like somatic experiences" (ELSEs). We catalogue the various ways in which ELSEs are described, how they are regarded by practitioners and teachers, the range of impacts associated with ELSEs, and the range of responses to these experiences recounted by meditation practitioners and meditation teachers.

\subsection{Previous Documentation of ELSEs}

Notions of physical or subtle body "energies" have circulated for centuries and across a range of cultural contexts. A comprehensive review of this literature would be well beyond the purpose and scope of this paper. Instead, we offer here a sketch of some of the different types of sources on energy systems and ELSEs in order to contextualize the unique contributions of the current project.

\subsubsection{Historical Background of Energy Systems and Associated Practices}

The main body of literature on energy systems is historical. This includes translations of key texts from religious, contemplative, and/or medical traditions on theories of the physical or subtle body, on the flows of subtle body "energies", or sometimes "winds", and on the relationship between the body and the world at large. References to subtle energies in various forms occur across a range of textual sources, from the Upanishads of ancient India, to classical Chinese medicine, to New Age spirituality. Samuel and Johnston (2013) provide a useful introduction to the range of materials available in the systems of Asian religions - such as the Yogic and Tantric traditions of Hinduism and Buddhism, the inner alchemy practices found in Daoism, and similar notions from Islam and Western Esoteric traditions. Many of these traditions have a long history of dialogue, exchange, or syncretism, both across religious systems and between religious and medical traditions, the latter of which also have comparable, although not necessarily identical, notions of the subtle body (Gyatso 2015; Zysk 1993). In Tibet, the medical and religious conceptions of subtle body energies operated independently but also in dialogue (Gerke 2013; Gyatso 2015). Tibetan medicine provides diagnoses and suggests treatments for various disorders in which subtle body "energies", or "winds" (Tib. rlung), can become imbalanced by numerous causes, including meditation practices (Benedict et al. 2009; Cayton 2007; Deane 2019; Samuel 2019). Tibetan Buddhist lineages also inherited from South Asian Tantric traditions a conception of subtle body energies that is soteriological in nature. Specific advanced contemplative practices, such as the techniques for the generation of inner heat (Tib. gtum mo), and the movements of the "magical wheel" (Tib. rtsa rlung "phrul 'khor), are understood to directly manipulate the subtle body energies through breathing, postures, physical movements, and/or visualization (Chaoul 2006; Cozort 1986). These practices have the proximate aim of purifying or removing various karmic obstructions to the subtle body by circulating the "energies" or "winds" (Tib. rlung) through a series of "channels" (Tib. rtsa) and "centers" (Tib. "khor lo), with the ultimate aim of effecting 
changes in consciousness thought to be more conducive to the attainment of awakening (Kongtrul 2007).

Despite myriad differences among the various systems of subtle body anatomy, and in the practical methods for engaging with subtle energies, most contemporary sources on this topic take as paradigmatic either the system of acupuncture and acupressure points through which energy (Chn. qi) flows in Chinese medical traditions, or the system of channels (Skt. nādi) and centers (Skt. cakra) that mediate the movements of breath, winds, or vital energy (Skt. prāna) according to South Asian Yogic and Tantric systems. Of particular significance in the latter systems is the idea that the female serpent goddess, kundalin $\bar{\imath}$, a manifestation of divine energy (Skt. sakti), lies coiled in the cakra associated with the base of the human spine. When stirred through Yogic and Tantric practices, this energy moves through the subtle body, and in particular up through the cakras associated with the central channel. These notions of the subtle body and the practice of stirring kundalini date back to Indic sources on Yoga and Tantra from around the 9th century CE (Samuel 2008; White 1996).

\subsubsection{The Emergence of Psychological Research into "Kundalini Awakening"}

The prominence of South Asian conceptions of the subtle body and subtle energies in New Age spirituality can be traced back to their popularization by the Theosophists in the late 19th and early 20th centuries (Albanese 2007; Hanegraaff 1996). Against this backdrop also emerged C.G. Jung's lectures on kundalini in the early 1930s and the prominence of the concept of "kundalini awakening" (Jung 1996). In the latter half of the 20th century, this idea was popularized in the West through the publication of Kundalini: The Evolutionary Energy in Man (Krishna 1967), and through the transpersonal psychology movement motivated by the work of Grof and Grof ([1986] 2017), who chose kundalini awakening as one of their paradigmatic examples of a "spiritual emergency". In their view, which is based largely upon Sannella (Sannella [1978] 1987), the phenomenology of a kundalini awakening is centered around ELSEs described as "powerful sensations of heat and energy streaming up the spine, associated with tremors, spasms, violent shaking, and complex twisting movements" (Grof and Grof [1986] 2017, p. 32), but also contains other behavioral, physiological, emotional, and perceptual changes. Research based on this conception has produced several scales assessing a range of anomalous phenomena (Goretzki et al. 2013; Greyson 2000; Sanches and Daniels 2008). For instance, physio-kundalini syndrome includes not just ELSEs, but also involuntary movements, thermal changes (heat and cold), somatic, visual, and auditory hallucinations, autoscopic dissociative experiences, intense positive and negative emotions, paralysis, and racing or slowing thoughts. Numerous case studies on experiences appraised as kundalini awakenings have been published. Some interpret the data as supporting a nonpathological model that acknowledges the reality of spiritual emergencies (Ossoff 1993; Taylor 2015), while other approaches attempt to establish a differential diagnosis with mental illness and present kundalini awakening as a culturally specific interpretive framework that patients might invoke to account for unusual experiences (Valanciute and Thampy 2011).

\subsubsection{Psychological and Medical Literature on ELSEs}

ELSEs have also been documented outside of the context of religious and contemplative systems and without the assumption that they are indicative of a kundalini awakening. Multiple ELSEs are part of a standardized taxonomy of experiences in schizophrenia spectrum disorders under the heading of "cenesthesias" (Gross et al. 2008; Parnas et al. 2005). "Migrating sensations" may be "fluctuating, wandering, circling or rising" throughout the body. Similarly, "sensations of movement, pulling and pressure" may be described as "vibrating", "quivering", or "simmering" among others. "Electrifying sensations" may appear as a "strange feeling, starting in the feet and radiating up to the head, like electricity", and can be "horrible" or "quite pleasant". "Thermic sensations" of heat, burning, or coldness may be "diffusely wave-like", "ascending", or "circumscribed" (Gross et al. 
2008, pp. 64-77). Cenesthesias may also be accompanied by emotions and/or emotional release. Cenesthesias typically fluctuate in intensity and may last seconds, minutes, hours, days, or even months or years. In a sample of first-episode psychosis patients, cenesthesias were one of the most common symptoms, reported by the majority of patients (67\%). Anomalous bodily experiences were significantly correlated with lower levels of physical activity (Nyboe et al. 2016).

\subsubsection{Qualitative Research on ELSEs}

A small body of literature has adopted a qualitative methodology to elicit rich descriptions of ELSEs and their appraisals, impacts, and associated responses. A recent study by Woollacott et al. (2020) used a mixed-methods approach with both open-ended qualitative data and survey-based quantitative data to capture descriptions of ELSEs and their impacts. Despite documenting a range of ELSEs and other perceptual and emotional experiences through their open-ended queries, the authors assume that all ELSEs are indicative of a kundalini awakening and a "spiritually transformative experience". As a consequence of these experiences, the majority of respondents reported either "minor increases" or "major increases" in a wide range of sensitivities, including across various modalities of sense perception, to the natural world, in interpersonal relationships, and to their nervous system. Nearly half of participants also reported an increase in sensitivity to mood fluctuations. The study did not assess whether these changes were associated with either positive or negative valences or impacts on functioning, although $50 \%$ reported an increase in overall health, while nearly $20 \%$ reported a decrease in overall health associated with the experience. Despite failing to clearly differentiate phenomenology from appraisals, the study offers a valuable assessment of the range of descriptions given to ELSEs, their co-occurring phenomenology, and the associated changes in beliefs and behaviors.

A different story emerges in research studies that investigate ELSEs within the context of meditation-related challenges. Buttner (2016) provides the results of a qualitative study of 12 teachers and 62 practitioners of Theravāda Buddhism that investigates the challenges or obstacles meditators face and how they resolve them. A total of 19 subjects (26\%) reported "disruptive anomalous energetic or kinesthetic experiences". The majority of subjects "found reframing the experience helpful, particularly viewing it as a sign of psychospiritual release or healing" (p. 179). A total of 10 subjects also engaged in self-regulation strategies, such as resourcing and body-oriented exercises, as a means of managing their ELSEs.

\subsection{Contributions of the Present Analysis}

The present analysis from the VCE study builds upon the existing literature in four significant ways. First, it documents ELSEs in a semi-structured interview on meditationrelated challenges reported by Western practitioners and teachers of Buddhist meditation across multiple lineages. In the context of the interview, ELSEs were not directly queried; rather, they were reported by participants only if they were salient to their narrative of meditation experiences. These reports reveal that meditation practitioners in the VCE study used a wide range of metaphors to describe ELSEs and their associated impacts. Second, the study does not presume that ELSEs are indications of kundalini awakening or any other interpretive framework; instead, by querying phenomenology independently of interpretations of meaning and value, this study shows that practitioners and teachers recruited a range of interpretive frameworks, from both within and beyond Buddhist meditation traditions, for appraising and responding to ELSEs. Third, in addition to interpretive frameworks, the VCE study also queried the range of responses and remedies, both helpful and unhelpful, that practitioners and teachers employed as a means of navigating ELSEs. These responses also tell us a great deal about the range of values associated with ELSEs, which can cut across medical, soteriological, and psychopathological discourses. Finally, because these experiences are reported in the context of meditation-related challenges, the study is able to address multiple impacts associated with ELSEs, including those that are 
ultimately described as leading to positive transformation, as well as those reported as distressing and impairing of functioning.

\section{The Methodology of the Varieties of Contemplative Experience Study}

The Varieties of Contemplative Experience (VCE) is a mixed-methods study of meditationrelated challenges, as reported by Buddhist meditation practitioners $(n=60)$ and meditation experts $(n=32)$ in the West (Lindahl et al. 2017). Through purposive sampling, male $(57 \%)$ and female practitioners, equally distributed among Theravāda, Zen, and Tibetan Buddhist lineages, were recruited to describe their meditation-related challenges, what they think caused them, how they and others interpreted them, and what remedies or responses were helpful or unhelpful for navigating them. Experts (meditation teachers and clinicians) were asked to describe meditation-related challenges they had witnessed in their students or clients, how they interpreted them, and what remedies or responses they suggested. A minority of meditation practitioners in the study reported a prior psychiatric history $(32 \%)$ or a history of trauma ( $43 \%)$. They had a wide range of meditation expertise at the time of the interview. Some were still novice practitioners, while others were advanced practitioners with more than 10,000 cumulative hours, many of whom were themselves teachers in their own meditation communities. The semi-structured interview protocol aimed to differentiate the descriptions of the meditation practitioners' challenges from interpretive frameworks, and, to facilitate this, participants were asked at the outset of the interview to recount their experiences without the use of tradition-specific terminology that serves as shorthand for, potentially, a range of experiences. In cases when practitioners initially employed tradition-specific terminology—-for instance, by first describing ELSEs in terms of kundalini, qi, or rlung - they were encouraged to attempt to present their experiences in descriptive terms and, later, to explain how they arrived at tradition-specific appraisals. A follow-up survey was also administered to practitioners to gather additional data on demographics, causality, influencing factors, and the effectiveness of remedies. The research methodology for this project was approved by the Brown University Institutional Review Board. For an additional description of the study methodology and demographics, see Lindahl et al. (2017). ${ }^{1}$

The interviews were transcribed and coded for phenomenology (59 categories across 7 domains), influencing factors ( 26 categories across 4 domains), and criteria for differential diagnosis or determining the need for intervention (11 categories). Overall results for the phenomenology and influencing factors are reported in Lindahl et al. (2017), and the results of the differential diagnosis analysis are reported in Lindahl et al. (2020). In the original study, the phenomenology category "somatic energy" was used to capture a range of ELSEs and was operationalized as "a type of sensation moving throughout the body or throughout a body area described with language of vibration, energy, current, or other related metaphors". Not included was any use of "energy" that referred exclusively either to an amount of strength, vitality, or arousal, or to metaphysical concepts in the absence of qualifying somatic phenomenology. ${ }^{2}$ This paper will use the term "energy-like somatic experiences" (ELSEs) throughout to highlight that "energy" was one of a range of metaphors and appraisals used by meditation practitioners and teachers.

ELSEs were the most commonly reported experience in the somatic domain, with $62 \%$ $(42 / 68)$ of practitioners voluntarily describing them. Nearly half of the experts $(16 / 33,48 \%)$ interviewed also mentioned working with meditators reporting ELSEs. ${ }^{3}$ However, as with most other types of experiences documented in the VCE study, not everyone who reported an ELSE found it distressing or impairing, as some practitioners qualified for participation in the study on account of challenges in other domains. Some discussion of ELSEs among twelve Tibetan Buddhists practicing Tantric or Vajrayāna Buddhism was previously reported in Lindahl (2017). The following sections provide a comprehensive investigation of ELSEs in the context of the VCE study by: (1) Cataloguing the metaphors practitioners used when speaking about them; (2) Describing the co-occurring phenomenology associated with ELSEs; (3) Detailing their overall trajectories and impacts; (4) Identifying the factors 
that influenced their nature or trajectory; (5) Documenting how ELSEs were interpreted and appraised by practitioners, teachers, and specialists, such as doctors and therapists; and (6) Explaining how practitioners responded to them or managed them with particular remedies.

\section{Communicating ELSEs through Metaphors}

\subsection{Metaphors Employed to Describe ELSEs}

A notable feature of practitioner descriptions of ELSEs was the frequent deployment of metaphors. Given the study methodology, which attempted as best as possible to differentiate phenomenology from interpretive frameworks, interviewers often directly prompted a redescription of tradition-specific terminology related to ELSEs. Practitioners regularly drew upon multiple metaphors in their attempts to describe their experiences, or to communicate different aspects of how ELSEs felt or impacted them. The following types of metaphors used by practitioners were identified.

\subsubsection{Energetic}

The most common way practitioners described ELSEs was, not surprisingly, as "energy" or as "energetic". To what extent these references are themselves metaphorical attempts to characterize an anomalous experience, and to what extent they reflect commitments to particular appraisals or metaphysical positions is often difficult to discern. Some practitioners explicitly distinguished their use of the term from a clear appraisal or metaphysical commitment. For instance, one practitioner described his ELSE as "energy-ish", while another declared, "I don't like the word energy, but it's hard to explain", before settling on "current" as his preferred metaphor. Numerous practitioners' depictions of ELSEs mixed energetic metaphors with other types of metaphors that will be detailed below. For instance, one Theravāda Buddhist also employed a hydraulic metaphor when reporting "waves of energy in the body [ ... that] would pass through my legs, my back, through my shoulders, out my arms". Other practitioners conjoined energetic metaphors with electrical metaphors by reporting a sense of "electrical energy" or feeling like they were "plugged into some energy outlet". The remaining sections emphasize the other metaphors beyond, or in addition to, "energy" that practitioners employed when attempting to describe ELSEs.

\subsubsection{Electrical}

The electrical metaphors used language such as "charge", "current", "circuit", and "voltage", in addition to making explicit comparisons with "electricity". One subject drew upon kinematic as well as electrical metaphors to describe his struggles to contain ELSEs: "I couldn't hold that energy. I couldn't run that much current. So it was like: What would happen to a toaster like that? It's kind of blown out". Others described more sudden surges, such as "a jolt of electricity from my heart to my head", or "bolts of electricity going up and down my spine".

\subsubsection{Vibratory}

Numerous practitioners used the term "vibrate" or "vibration" when describing ELSEs. One Theravāda Buddhist practitioner recounted: "I went to my bed to go to sleep, and I remember falling asleep just feeling this warm, fuzzy feeling. And then I woke up around like two hours later-I think I did an hour of meditation, actually, before going to sleep-and I woke up, and I felt my entire torso vibrating-like, on the inside". When prompted to give an account of what she meant by "energy", a practitioner in a Tibetan Buddhist tradition said she "equated it to standing next to a 747 as it's firing up its engines on the end of the runway. It felt like that much vibration energy". Another Tibetan Buddhist practitioner drew upon a very similar example to describe "flows of energy or heat, vibrations", saying, "if you put your hand in front of the exhaust when the car engine is running, it's a bit like that sometimes". 


\subsubsection{Hydraulic/Pneumatic}

Hydraulic metaphors reference water (e.g., a wave, a rip tide, a river) to help characterize ELSEs, whereas pneumatic metaphors reference air (e.g., wind, draft). However, there is also a high degree of overlap between these references because they relate to the dynamics of liquid or gaseous matter in similar ways. For example, "vortex", "flow", "build-up", "channel", "fizzing", "effervescence", and similar words used to describe ELSEs, have pneumatic as well as hydraulic associations. One Zen Buddhist reported "the sense that I can open up my hips and that the energy flows through-that it doesn't get stuck. And I have a tendency to stick and to reverse the flow, because there is a natural flow. And I have this unconscious ability to sort of reverse the flow". Here, the hydraulic/pneumatic metaphor of "flow" overlaps with his conceptualization and regulation of ELSEs that were initially challenging, as articulated through the use of a "blockage" metaphor, which will be addressed below.

\subsubsection{Kinematic}

Other metaphors drew on the processes of kinematic physics, and, in particular, the dynamics of solid extended objects. These included metaphors of "bursting", "hitting", and "tearing". One Theravāda Buddhist practitioner, for instance, described how "going up my spine there were these energetic bursts". Another practitioner combined kinematic and hydraulic metaphors in an evocative example, stating "if you have a riverbank and [ ... ] there are rapids going by, you know how the riverbank will fall into the water? It kind of felt like that. Like this riverbank is running and my muscles were being kind of torn at".

\subsubsection{Blockages}

Participants occasionally made use of metaphors that conveyed impediments or obstacles, such as when the "energy could start to knot". As illustrated above, blockage metaphors were also often contrasted with hydraulic/pneumatic metaphors that characterized energetic "flows". They also were deployed in contrast with kinematic metaphors, with one Zen practitioner describing how she "could feel [the energy] beating against any blockage until it would either burst through or I would scream mercy".

\subsubsection{Combustive}

The practitioners also used metaphors that incorporated elements of combustion. These included "burning", "fire", and "exploding", among others. It is likely that, in some instances, combustive metaphors gestured towards co-occurring changes in perceived body temperature, which were not uncommon. One practitioner described an ELSE as "almost a feeling like something is going to explode or wants to explode". Another reported: "it was like electricity; I felt I was on fire".

\subsubsection{Mechanistic}

These metaphors drew on machines and mechanical systems to express aspects of ELSEs. One practitioner explained how she began "experiencing incredible upward energy in meditation" that "was like being a rocket ship [... ] Like that, except all the stuff was going up". Another practitioner likened his experience to feeling "like a machine with gears-it felt like, all of a sudden, all the gears started to grind. [ ... ] The sensation was of just this horrific, grinding discomfort". Notably, gears can operate smoothly, or they can grind, which for this practitioner was a means of communicating dysfunction.

\subsubsection{Spatial/Movement}

Some metaphors conveyed spatial relationships or served to locate experiences in an embodied or conceptual space. Spatial metaphors were often closely tied to metaphors that communicated movement. One practitioner described how, in her experience, "energy [ ... ] all built back up in the body again and went down again, over and over and over again". Another practitioner described how, for her, it was "like a vibration cascading through the 
body. [ ... ] Sometimes it can go up; sometimes it can be coming down". Some movement metaphors denoted a kind of progression within the body, as for one practitioner who reported that "energy [ ... ] advances from one vertebra to another". Notably, paradigmatic texts on kundalini typically characterize it as progressing upward from the base of the spine. Other practitioners used a spatial/movement metaphor of "opening" to contrast with constrictions or blockages.

\subsubsection{Agentive}

Finally, some metaphors employed concepts that pertain to agents or agency, often appearing to attribute volition, initiative, and control to ELSEs. These metaphors were often coupled with a grammatical shift, where the participant was in the position of the object, while the ELSE was presented as the subject. Some uses of agentive metaphors attributed initiative to the "energy" or to attendant phenomena. One practitioner stated, "it just sort of does what it wants to do", while another explained, "it's like wiggling through your body, and it's trying to get through the flesh, and you can feel the energy moving through your body and even through your brain all of the time". The most pronounced exemplars of these metaphors referred to the "energy" as a separate entity with its own agency: "I was sharing my physical body and my life force with energy that I felt very clearly didn't belong to me or emanate from me. And by ' $m e^{\prime}$ I mean the self that I had previously understood as the ordering principle of who I was".

\subsection{Functions of Metaphor in Communicating ELSEs}

Research suggests that rich relationships exist between metaphors, on the one hand, and the ways in which people explain and respond to phenomena, on the other. Metaphorical language may be especially important when people attempt to make sense of experiences or ideas that are novel or complex, or that otherwise make comprehension challenging (Landau 2018). Daniel (1994) observed, for example, that the recovery from experiences of extreme duress is accompanied by a gradual increase in metaphor use. Narrative psychology has long noted the presence and utility of metaphors within "recovery narratives" (Hydén 1995; Llewellyn-Beardsley et al. 2019). One way that metaphors may ease comprehension and communication is by applying the knowledge, heuristics, and associations of something familiar to something that is less familiar. Metaphors are also closely tied to cultural contexts, as they draw upon broader enculturated discursive vocabularies. Cultural environments may afford different kinds of metaphors (Musolff 2017), and even different degrees of preference for metaphor use overall (Ondish et al. 2019). This can extend to the meaning and interpretation of unexpected or challenging experiences. The application of a hydraulic metaphor, such as "flow", may accompany interpretations (such as $q i$ ) that employ similar language, as well as responses (such as acupuncture) that treat the ELSEs in terms of blockage, release, and other means of regulating flow. Furthermore, certain energy systems are becoming more widely known in the West as, for example, acupuncture and yoga become more mainstream, and various iterations of spiritual emergency frameworks become part of the discourse on mental health and spiritual experience.

\section{Co-occurring Phenomenology}

ELSEs were not typically reported as occurring on their own as an isolated or circumscribed experience. Rather, multiple practitioners and teachers identified ELSEs as having caused, or otherwise been linked, directly or indirectly, to a range of additional challenging phenomenology.

One Tibetan Buddhist practitioner reported a number of other somatic phenomena cooccurring with ELSEs, many of which were mentioned by other practitioners in the study as well. Her relentless experiences of "energy" led to "intense heat", including a sensation of "fire coming out of the top of my head", as well as involuntary movements, such as "whole-body jerking", and "spontaneous [ ... ] back-bending". These "energy flows" could 
also be "extremely painful". In one instance, she had "energy [ ... ] rising up out of my pelvis and going up through my chest", and she "felt like there was a dagger or something embedding itself in my heart from below". She also reported the "energy" concentrating in her head, producing feelings of pressure, and found herself unable to get it to "distribute more uniformly" through her body. At its most "intense", the "energy" felt "electrical" and seems to have caused "really strange" phenomena, such as "when I would pick up my phone and it would freeze", or "my computer would freeze". With a constant sense of "energy" flowing through her body, she also experienced difficulty sleeping. Beyond these somatic effects, there were also affective and cognitive consequences, such as having "a lot of anxiety" because of her discomfort, uncertainty around what was happening, as well as "trouble focusing" because of the constant and unusual nature of her body sensations. A Theravāda Buddhist teacher associated "feeling a lot of energy in the body" with "a rapturous sense, [ . . . ] feeling like your circuits are gonna get blown out, a lot of sexual kind of energy, not sleeping - sometimes not sleeping at all-feeling like you can't sleep, weird dreams, vivid dreams, a lot of sexual thoughts, shaking, [ . . ] involuntary movements".

Certain combinations of somatic and cognitive changes also proved to be significant. One Tibetan Buddhist practitioner experienced an ongoing feeling of "rlung" getting "stuck" in his "heart center", creating an intense feeling of "constriction" in his chest, such that it felt it was "between two metal plates" that had been "squeezed together". With the energy unable to "flow properly in the rest of the body", he felt "wired, like having drunk 50 cups of coffee", and had a "racing" mind "like a whirlwind". "Dizzy" and "unable to concentrate", his condition also interfered with his sleep. According to a Tibetan Buddhist teacher, when "the energy in the body begins to rise", it is a "classic sign of [things] going wrong", and causes practitioners to "get very speedy, flighty, airy. They can't remember things very well. They get a little bit jumpy".

Affective changes were often noted in association with ELSEs and ranged in valence from strong positive affect to intense fear. ELSEs were sometimes accompanied by reexperiencing or uncovering traumatic memories, which some teachers attributed to a process of "opening up" to that which is "stored in the body". Immediately following the onset of ELSEs, one Tibetan Buddhist practitioner reported having four months of "very gentle [ ... ] euphoria". Then, on a retreat she experienced vivid imagery related to a childhood trauma, which she described as "beautiful" and as having left her "more embodied" and "less dissociated". Over time, this had the effect of "opening up [... ] all the trauma of my life", and this was followed by affective and cognitive changes that proved to be very challenging, such as an escalation of positive affect into "mania", feelings of paranoia, and a frightening sense that "my body was being controlled", eventually culminating in multiple hospitalizations for what she described as psychosis.

In some cases, ELSEs were also associated with perceptual changes. A Tibetan Buddhist practitioner experienced a visual component to his ELSEs. He described a "vortex of white energy light forming out of the [...] crown of my head" and "breaking through the crown cakra". Subsequently, he reported a "visual hallucination" of numbers on a cable TV box that he interpreted, in his altered state, as "heralding my enlightenment". A Theravāda Buddhist practitioner recounted an instance in which an ELSE was associated with specific visual changes:

the field of my vision would soften and this very deep, deep blue--kind of an indigo blue-would start to take over the field of my vision, so everything I was seeing would start to fall away, and this blue would start to fill the space and intensify and, as it intensified, the energy would be rising up in me. [ ... ] It was like the blue is intensifying, everything else is disappearing. I'm feeling as if I'm about to disappear. 


\section{Impacts and Trajectories of ELSEs}

\subsection{Time Course}

For most of the practitioners who reported them, ELSEs had a significant or central impact on their overall trajectory. The time courses for ELSEs typically fell into one of three patterns: transient, recurring, or enduring. Transient ELSEs were often confined to a single day or a single retreat. A Theravāda Buddhist teacher said that, of his retreat participants who get "stuck on" ELSEs, "most always it will calm down. [ . . . ] [In] twenty-four hours, it usually runs its course-some people, two or three days". Transient ELSEs were sometimes intense but, in themselves, did not often have a large impact on the lives of our participants. However, in some cases, transient ELSEs occurred at the very beginning of the onset of challenging phenomenology, the course of which could be quite severe and impairing.

Recurring ELSEs were often associated with periods of retreat or intensive practice. One Zen Buddhist practitioner first experienced "energy" on a retreat and found it challenging to manage until she found a teacher who offered her a series of remedies that she could apply. In the 33 years since, ELSEs have continued to occur each time she does retreats, but they never persist into daily life. The difference has been that she now has tools to work with the "energy" and so, rather than being uncomfortable and distressing, it moves in a way that is gentle and that aids her practice and dissipates when the period of intensive practice ceases.

Enduring ELSEs are those that continue, more or less uninterrupted, from meditation practice into daily life, often lasting for many months or years. Some practitioners described "constant" ELSEs, including during sleep, that went on for decades. One Theravāda Buddhist teacher suggested that the "kundalini process" can take as long as 12 years. Such cases may require significant effort to work with ELSEs and their co-occurring phenomenology, especially when there is ongoing associated distress and/or functional impairment.

\subsection{Positive and Negative Impacts Associated with ELSEs}

In addition to the variation in the time course and frequency, ELSEs also unfolded along a variety of trajectories. Given that the VCE study aimed specifically to investigate meditation-related challenges, many reports of ELSEs were presented as such. However, for some practitioners, ELSEs were exclusively positive, and, in other instances, they unfolded according to mixed trajectories that had a combination of positive and negative dimensions.

For some practitioners, ELSEs started positive and remained positive. One Zen practitioner experienced a "joyful" feeling of "energy" rising up his spine. As it reached his "upper-middle back", he experienced a "heavy vibration" accompanied by significant changes in his sense of self: "I know that that moment when I felt that vibration that something basic in my brain chemistry changed and that's really when [practitioner's name] - the [practitioner's name] that I knew-sort of disappeared. He was gone". He described this experience as "sort of a death of self", in which he had been "replaced by the rest of the universe", which he likened to "a great circuit of energy". His teacher identified this as a transformative awakening experience. Following this breakthrough, he began to notice an ongoing "presence of energy at the base of my spine" that he could "draw up", after which it would go "out the top of my head into the universe", which he described as feeling "really good". For this practitioner, the sense of "energy" was not circumscribed to his body, but instead connected him to the "circuit" that extended into the "vast universe". He subsequently became a teacher in his Zen tradition, and his experiences of "energy" continued to fill him with a sense of "wellbeing". They also aid his teaching because when he's "expounding the Dharma, it's coming from that place at the base of my spine".

For some practitioners, what began as a positive ELSE became negative in its impact over time. After a Tibetan Buddhist practitioner's initial ELSE, he "was just left in a state of profound awe. Literally jaw-dropped type of experience". However, soon, "it was starting to go into some wrong channels and it was just excruciating". In addition to pain, the 
ELSE also caused a kind of hypersexuality, which led him to "behaving in a way" that his partner "was unaccustomed to" because of the "intensity" — something that was "scary" for his partner and "unpleasant" for him. Another Tibetan Buddhist practitioner on a long-term solitary retreat was engaged in subtle body practices, such as the cultivation of inner heat, which started off with anticipated positive experiences of "energy rising up, heat coming up, and cool, blissful, white radiant showers coming down". Later, in the retreat, the movement of "energy" changed such that she "started to feel energy running out of my body [...] If you held your hand between my legs, it was like you could feel energy spilling onto your hand. [...] I called this feeling 'draining'. [...] It really just felt like bleeding to death". She tried various movements and sleeping in twisted postures in an attempt to stop the "draining", but ultimately did not obtain relief until her teacher took her out of retreat and she sought sessions with acupuncturists and energy workers to address the "draining".

Other practitioners had ELSEs that began as, and remained, negative. A Tibetan Buddhist practitioner had his first ELSE during a retreat and found it "very disconcerting" and "very traumatic". He began to "wonder what the hell is it, and is this is supposed to be healthy, or is this a problem?" As it persisted, it also led to additional challenges, such as heat, neck tension, and insomnia. Drawing upon one of the frameworks used within his lineage, he characterized his symptoms as an instance of a "rlung disorder". In the 11 years since, he has had to work constantly to manage these symptoms, using a variety of remedies, otherwise it "gets really, really unpleasant" physically.

In some cases, practitioners initially found ELSEs to be negative, but over time had a shift (in understanding, appraisal, or through a change in phenomenology) towards a positive experience. One Zen Buddhist practitioner initially struggled to work with and make sense of ELSEs that would occur when she was on retreat. In the beginning, she found the ELSEs "physically uncomfortable" on account of feeling "like something was being shaken inside". One instance led to a temporary paralysis of her legs, which she described as "the most dramatic experience I had with kundalini". However, after learning to manage the "energy", it began to have a positive and transformative effect on her life. She reported "energetic" build-up and release, accompanied by the resurfacing of intense emotions related to past experiences. This "would start as a pain somewhere in my body and, when it would release, there would be a story that would release with it". As the story and the associated emotion released, there would also be "energy in my body that would open and release", and the story would become "resolved". She described how "certain mindsets that I had got broken up" by "kundalini", with the result that she "understood things in different ways about both my life and my relationships and about other people and my own history", which was "very helpful".

\subsection{Functional Impairments Associated with Challenging ELSEs}

Some degree of impairment was not uncommon among practitioners reporting challenging ELSEs. The types of impairment caused by ELSEs, or by co-occurring phenomenology, cover a wide range, from physiological to behavioral. Impairments also ranged from mild to severe and could be transient as well as enduring. Impairments occurred in many areas of life, causing challenges with day-to-day tasks (such as difficulty driving), or problems maintaining relationships or employment.

One Zen practitioner, previously a performing artist, became unable to work because of the "extremely troubling" experiences of "energetic updraft" — "too much energy, that my body couldn't hold" - that would occur during her performances. An ongoing involuntary "yanking" of her body in one direction was also associated with her ELSEs, which left her unable to sit comfortably for a year. In addition, "trauma material came up" and she became "very, very emotionally volatile". She explained:

lots of people have energetic experiences where they feel voltage. Lots of people have trauma memories come up. That's not unusual, that's a part of the practice. 
So why are some of us so destabilized and really have difficulty coping with that and recovering from it, and why do other people kind of move through it?

She suspects the reason was "because I was so energetically destabilized, that when the trauma material came up, as it does for most people who have trauma in their backgrounds and who have tried these meditation practices, hopefully the stuff is gonna clear-you're gonna go through life and kind of clear it—-but because of the energetic destabilization, I just got more destabilized".

A Theravāda Buddhist practitioner found that ELSEs would build in his body so intensely and so persistently that he needed to find ways to regularly "release" them. He was only able to remain functional at work by taking hourly breaks of twenty minutes to go onto the rooftop and "just scream and shout and move and just let all this energy dump out into the loud city where it would mingle and disappear". A Zen Buddhist practitioner described five years of "energy [ ... ] running through you day and night and you can't escape it", as well as extensive associated phenomenology, including insomnia, involuntary movements, and significant changes in her sense of self. Her ongoing experience of trying to manage these unrelenting ELSEs and the associated phenomenology became so allconsuming that, for a time, she was unable to care for her school-aged children. Eventually her marriage ended, with her husband saying, "come and see me when you're back to normal".

In a number of cases, insomnia caused by ongoing ELSEs was associated with functional impairments, or with additional challenges appraised as mania or psychosis. A Theravāda practitioner undertaking her first retreat began experiencing "so much energy running that I [ . . ] didn't feel the need to eat or sleep. It didn't occur to me. [ . . . I I wasn't tired at all". She soon "lost track of linear time" and began having visual changes where "things that would appear as solid objects didn't seem so solid anymore. [ ... ] It was almost like I was seeing energy instead of just seeing solid objects and believing that they were totally solid". Her behavior began to deviate from the protocols of the retreat and got the attention of a teacher, who tried to speak with her and found her nonresponsive. When she was finally able to speak, she asked for guidance for managing "the sensation of tons of energy shooting through my hands", something that "sort of freaked me out" because it was "really strong". Mainly because of her nonresponsiveness, the teacher decided to remove her from the retreat and had an ambulance take her to the hospital. She was admitted against her wishes to the psychiatric ward upon telling the psychiatrist that she was "seeing energy again: looking at people and seeing their energy body, seeing energy flow through things. [ ... ] And so he thought I was nuts and decided to keep me there". She was treated with "heavy-duty [anti-psychotic] medications" in an attempt to get her to sleep. She expressed frustration at receiving this treatment when, in her view, she was "seeing a lot of things psychically", whereas the doctors "think I'm crazy; they're treating me like a crazy person".

\section{Factors Influencing the Onset and Trajectory of ELSEs}

The practitioners and experts identified a number of influencing factors they associated with the occurrence and trajectories of ELSEs. The meditation practitioners most frequently attributed the emergence of ELSEs to the type of meditation practice they engaged in, particular approaches to their meditation practice, or to the amount (e.g., hours, days, etc.) or intensity (e.g., effortfulness) of meditation practice. Practitioners and experts who identified the amount or intensity of practice as influencing factors referred specifically to an increased amount or intensity. An increased practice amount, in the context of a meditation retreat, was highlighted as a factor. In some cases, it was noted that this was the practitioner's first retreat, or that it was a longer retreat than they had attempted before. One practitioner accepted her ELSE and the co-occurring involuntary movements as normal (though "bizarre") after one of the teachers noted in a talk that, on retreat, "sometimes your body would do really strange things" and you just need to "observe it all". Many practitioners who identified a meditation practice approach as a factor specified that 
it was an effortful, intense, or striving style of practice that led to the ELSE. One described his approach, for example, as "pushing and pushing" - that of an "overachiever" who was "just super trying to do it right". Another had been drawing on intense "willpower" to "grin and bear it" through intensive practice prior to the onset of challenging ELSEs.

Unsurprisingly, meditators whose practices intentionally engage with ELSEs, and whose practice tradition provides a framework for understanding them, more commonly reported the type of practice as an influencing factor. Specifically, these were Tibetan Buddhist practitioners engaged in Vajrayāna practices that involve working with the "channels" and "energies" of the "subtle body" (see also Lindahl (2017)), and Theravāda practitioners in S.N. Goenka's vipassanā tradition, which emphasizes observing somatic sensations. Although practitioners in traditions with subtle energy frameworks as a component of the meditation practice instructions showed a greater tendency to identify practice type as an influencing factor, these practitioners were not more likely to report ELSEs than those from other traditions that did not have energy models. Even though a similar number of Zen Buddhist practitioners (10/20) and Tibetan Buddhist practitioners $(13 / 20)$ reported ELSEs, not a single Zen practitioner identified their type of practice as an influencing factor, whereas 77\% (10/13) of Tibetan Buddhist practitioners did. This was also the case for Theravāda Buddhists practicing outside of the tradition of vipassanā as taught by S.N. Goenka. While 75\% (6/8) of the practitioners of Goenka's vipassanā identified the type of practice as an influencing factor for ELSEs, among Theravāda Buddhists practicing in other lineages, only one in eleven that reported ELSEs identified type of practice as an influencing factor. One possible explanation for this discrepancy is that Zen Buddhists and Theravāda Buddhists outside of Goenka's tradition do not employ frameworks for understanding and working with ELSEs to the same extent, nor do they engage in meditation practice approaches oriented towards intentionally discerning or engaging with ELSEs.

Some influencing factors were identified as having aggravated, if not directly caused, ELSEs, making them more intense or more difficult to manage. These include reduced sleep or appetite, having a trauma history, having life stressors (such as work-related stress, grief, or relationship problems), having an inappropriate diet (especially vegetarian), and a lack of prior training or preparation related to the "subtle body" - one practitioner described herself as not having developed the "wiring" required to safely manage the emergence of "energy".

Less common, but notable, were occurrences where the associated catalyst for the ELSEs was located outside of the meditator. For instance, one practitioner understood his ELSEs as the result of being in the presence of a "powerful" teacher. Another Tibetan Buddhist practitioner, who had undertaken multiple three-year retreats, associated ELSEs with simply being in a retreat center. He described feeling "electrocuted" at a "low-level" upon entering, which remained "constant" as long as he was in the retreat location.

\section{Interpretive Frameworks Recruited for Making Sense of ELSEs}

\subsection{Engagements with Interpretive Frameworks}

As with other conceptually mediated phenomena, one is not likely to appraise a phenomenon specifically as "energetic" without energy-related concepts. Thus, interpretation may, from the outset, play an important role in practitioners' ELSEs. The presence or absence of frameworks, the quest for them, and attitudes toward them were often salient features of the meditators' narratives.

Meditators sometimes found that they did not possess an adequate interpretive framework to enable them to manage their ELSEs at the time they occurred. For example, one practitioner in a Zen lineage remarked that she initially "had no idea what to make of" her ELSE. The process of acquiring workable interpretations for ELSEs often involved other people. Meditation teachers, therapists, or healers providing both alternative and allopathic medical care could play an influential role in offering a framework for under- 
standing ELSEs, especially when those frameworks were not a part of the meditator's prior contemplative training.

In a few cases, meditators highlighted the close fit of an ELSE with existing frameworks, referring to the ways that their experiences were prototypical of certain interpretations, such as kundalini. When there was a sense of fit between the phenomenology and a framework, the experience was often interpreted as either confirming the validity of the framework, or at least as benefiting from the framework's utility for meaning-making and determining appropriate responses. For example, one meditator stated: "I had, at one point, a very classic kundalini experience with feeling the energy down that main core of the body, and I was so surprised by it. I thought, 'Wow, it's actually true'!" By contrast, the perceptions of poor fit were consistent with the rejection, or incomplete adoption, of a framework. Another meditator recounted, "I found lots of people with lots of advice and conceptual boxes. [ ... ] But I never found, in all of my experience, anybody who just could speak that energy language and somehow engage with it in a helpful way".

\subsection{Interpretations Aligned with Buddhist and Other Religious Traditions}

Some practitioners reported previously held beliefs in established frameworks related to ELSEs, such as notions of spiritual emergency or kundalini awakening, of psychosomatic or karmic purification, or of a subtle body in general. Others learned about these frameworks from teachers or fellow practitioners in their communities in the context of responding to, or making meaning from, ELSEs. Religious frameworks also sometimes posited that ELSEs could arise at particular stages of contemplative practice. In such instances, ELSEs could still be considered normative (that is, experiences that should arise at a particular stage or through practice), as normal-but-not-normative experiences of little overall significance, or even as unwanted side effects of practice.

\subsubsection{Theravāda Buddhism}

Most lineages of Theravāda Buddhism did not provide frameworks relating to the subtle body or subtle body energies, although a few Theravāda Buddhists did apply other tradition-specific terminology to their ELSEs. One practitioner associated his ELSEs with "access concentration" (a sustained high level of focus), and one Theravāda teacher thought that ELSEs could be indicative of an excess of piti-a Pali term commonly translated as "rapture", and which is a quality expected to arise through the cultivation of concentration. Physical experiences of rapture, including "sudden jerks" and "excitement that spreads through the whole body [and] seems to undulate in the body like the waves of the sea", are also listed among the "ten corruptions of insight" (Pali vipassanā-upakkilesa)_anomalous perceptual and emotional experiences that are thought to potentially sidetrack meditators' progress if they mistakenly take them to be indicative of a genuine meditative attainment (Sayadaw 2016, p. 371).

Nevertheless, most practitioners of Theravāda Buddhism in the VCE study did not encounter adequate frameworks for how to appraise ELSEs from within their tradition. Thus, they often engaged frameworks from other traditions. For example, one practitioner described how her experience of "energy" mapped onto conceptions of the subtle body comprised of nādis and cakras, as found in South Asian Yogic and Tantric systems. Another practitioner, on a multiweek retreat, reported that while she was meditating on her bed, she felt "energy come up into my body and bang my back against the wall", which happened "four or five times and then my head got thrown backwards". She didn't know how to interpret the experience, which she found "pretty dramatic". When she reported her "convulsions" to her teachers, they removed her from the retreat without giving her a framework for making sense of what had happened. Instead, she was referred to a psychiatrist. A therapist she later consulted immediately appraised her experience as a "kundalini release". Excited that she had a framework for understanding what had happened to her on retreat, she went back to the center and found the teachers she had worked with, saying, "“This is what happened to me: I had a kundalini release'. It was like, 
'I know now'! I was like an excited little kid-'I know now'! And they said they don't believe in kundalini releases".

Among Theravāda Buddhist practitioners, one group with existing frameworks for ELSEs were those in the tradition of vipassana meditation as taught by S.N. Goenka, who tended to describe their ELSEs in terms of "subtle sensations". This is consistent with meditation practice instructions in this tradition, which include language focused on sensations, tingling, and vibrations as cues for what to notice during the body scan practice in particular (Goenka [1987] 2000). One practitioner described her experience with the body scan as initially including "tinglings around all of my body. It felt like-it wasn't electricity; it was more of like very pleasant tinglings". She described this phase as being in accordance with reaching a "free-flow experience" with the body scan.

\subsubsection{Zen Buddhism}

One practitioner, for whom ELSE problems occurred in conjunction with other somatic phenomenology, had his experience appraised as a form of "meditation sickness" (Chn. chan bing), which, in Chan and Zen traditions, refers to a range of meditation-related obstacles, challenges, or incorrect practice approaches (Ahn 2021; Greene 2021). More typically, however, practitioners in Zen lineages did not make use of Zen-specific terminology for ELSEs and described there being few emic frameworks available to them. One Zen practitioner described "floundering [ ... ] a little bit" as her teacher struggled to make sense of her ELSEs. "He was a little distressed and he didn't quite know what to do with me". One Zen teacher acknowledged, "We don't talk about [ ... ] these physical manifestations [ ... ] much in Zen". As with some Theravāda practitioners mentioned above, some Zen practitioners sought frameworks and methods for working with ELSEs from outside of their tradition. However, Zen practitioners often referred to a Zen approach toward relating to anomalous and unexpected experiences in general, which was also applied toward ELSEs. This approach entails regarding unusual experiences as insignificant at best, and potentially distracting or derailing at worst, and, thus, involves de-emphasizing them in interpersonal communication, attributing them minimal significance.

\subsubsection{Tibetan Buddhism}

Intentionally working with the "energies" or "winds" (Tib. rlung) of the subtle body can be a dimension of advanced contemplative practice, especially practices associated with Vajrayāna or Tantric Buddhism, such as subtle body yogas or completion-stage visualizations (Cozort 1986). Many Vajrayāna practitioners worked within that framework, some despite degrees of distress or discomfort associated with the subtle and/or physical body. For an in-depth discussion of experiences and appraisals reported by Tibetan Buddhists engaged in Vajrayāna practices, see Lindahl (2017). In other practice contexts in Tibetan Buddhism, such as the cultivation of śamatha (calm abiding), ELSEs were appraised as transient meditation-related side effects (Tib. nyams) that are not the goal of practice, but which can be an indication of progress in meditation (Lingpa 2015). One practitioner of śamatha recounted how "huge amounts of blissful energy would overtake me". When she described this to her teacher, he replied by stating, "Don't worry about it, don't pay attention to it-it's just a nyams".

\subsubsection{Purification}

Practitioners across Buddhist traditions sometimes associated the presence of ELSEs with a process of purification. Notions of purification can be found across Buddhist traditions and typically refer to the elimination of the influence of karma, and the eradication of cognitive and affective deficits that "obscure" the mind (Sferra 1999). In Vajrayāna Buddhist traditions, the basis of purification is sometimes presented as being the subtle body. For instance, according to Kongtrul (2007), the subtle body's "energy currents [ . . ] ] are the contributing condition for igniting inner heat", and that inner heat (Tib. gtum mo) is described as purifying through the metaphor of "burning" on account of how "when it is 
ignited the habitual patterns of the aggregates, constituents, and sense fields are burned up" (p. 168). Numerous VCE study participants from this tradition also described how the obscurations, attributed to karma more generally, or to psychological or emotional tendencies in particular, manifest as "knots" in the subtle body (see also Lindahl (2017) and Rinpoche (2012)). A Tibetan Buddhist teacher explained that, initially for the meditator, "everything is twisted and knotted up and the winds don't turn properly [ ... ] The purification process literally takes place within the subtle body", a process in which "these knots [ ... ] break loose", with the ultimate goal of becoming "an enlightened being, or a realized being" for whom "the channels are translucent and straight, and all the energies therefore flow through them".

Some practitioners of Goenka's vipassanā understood their experience along similar lines. The same practitioner, who above described having a full-body tingling that she associated with being in a "free-flow" state during the body scan practice, also described a more dramatic change that soon followed. As she continued her practice, she recounted how her arms and elbows became involuntarily contorted into an unusual position and frozen. Then, "there was an intense rush, block of electricity, and it was like moving throughout my hand and leaving my fingers. And once this electricity left my fingers, I was able to move it again". When she spoke with a teacher about this, the teacher said "'these are old sankhäras leaving you'. She specifically said that they leave as violently as you repress them". In this approach, the ELSEs and their physical manifestations are interpreted as indicating a disruption in the influence of sainkhäras - or mental formationsone of the five psychophysical aggregates influenced by karma. Other practitioners in this tradition also drew a connection between ELSEs, karma, and notions of purification:

On the whole and looking back now, I feel it was stuff from the past just purifying, just trying to come out. And that's what it is when I experience it on retreats now. It feels very much like it's just energy from the past unlocking itself, that's kind of tight and clenched and held in the body, and it just kind of unlocks itself, and flows. The unblocking of energy.

Emic understandings of purification may not always be easily separable from more generalized culturally salient notions that the practitioner was previously in a less ideal state, and that challenges encountered in and through meditation are part of an ultimately ennobling personal trajectory, such as those characterized elsewhere as "redemptive" narratives (McAdams et al. 2001; Palitsky et al. 2019). These narratives can be typified by language suggesting personal progress, growth, development, and even individuation. For example, despite his skepticism about subtle body systems, one Tibetan Buddhist practitioner still felt aligned with notions of "energetic" purification and a progressive trajectory of personal development:

I just have to say, I hate cakras. I'm not interested in cakras. I kind of get this aversive quality around New Age-y people who talk about their cakras. I've never been interested in this shit. I still don't care about it. I never got caught up in it. But I also cannot deny the reality of my experience. [ . . . ] So, do I think that the deep concentration practices were causing either a purification or kind of helping get this energy moving up my spine? Yeah, absolutely. Did it finally, kind of, break through the crown cakra? Yeah, I think that's exactly what happened. And I think it's exactly what needed to happen. [ ... ] It just felt my body was energetically purifying itself to be able to do this kind of deeper meditative, spiritual work.

\subsubsection{Kundalini}

Some practitioners from all three traditions of Buddhism also looked beyond the frameworks intrinsic to their lineage. As mentioned above, in some cases, this happened on account of a perceived absence within the practitioner's tradition of suitable frameworks to match or account for their experiences. Overall, kundalini was the most commonly used interpretive framework for describing ELSEs by practitioners in this study. Given 
that the notion of kundalini can be found in Buddhist Yogic and Tantric traditions, in the broader context of South Asian religions, and in the contemporary spiritual emergency framework put forth in transpersonal psychology, it is often difficult to know which framework (or combination thereof) practitioners had in mind when invoking this term as an account of their ELSEs. Some practitioners used kundalini to typify intense ELSEs, which involved somatic components, were deemed risky, included a notion of "energy" progressing through the physical or subtle body (usually up the spine), and perhaps were accompanied by clusters of key co-occurring phenomenology identified in the spiritual emergency literature, such as heat and involuntary movements. This was especially the case for practitioners with previous knowledge of what kundalini phenomena may entail, and who recognized their ELSEs as such. One Tibetan Buddhist practitioner declared: "what happened to me was a profound kundalini awakening. I'm absolutely certain of that. [... ] All of what I read about kundalini is that that's exactly how it shows up".

However, in a number of practitioners' narratives, kundalini appeared to be a more general label associated with disruptive and transformative ELSEs. Some of these practitioners had experiences that did not necessarily follow the normative paradigm, but which were nevertheless appraised as related to kundalini. For instance, one Theravāda Buddhist practitioner described his experience in terms of a kundalini awakening event despite a lack of fit in some respects:

I did understand that a kundalini awakening was the best approximate map for what was going on. But at the same time I also realized that in my case the map was only partially helpful. Because, for instance, it talks about the energy rising up through the cakras, but in my experience it was always chaotic - it would be here and there and there was never any sense of progression.

It was not uncommon for practitioners to find themselves in the midst of ELSEs, only to come upon the kundalini framework afterwards. Sometimes practitioners saw the kundalini framework as only a partial fit, and, as will be addressed below, this appraisal could co-occur with other appraisals.

\subsection{Interpretations Drawn from Medical and Scientific Traditions \\ 7.3.1. Traditional Tibetan Medicine}

As explained above, although "energies" or "winds" (Tib. rlung) are intentionally engaged in the context of some Tibetan Buddhist practices, and in other contexts are treated as an insignificant "meditation experience" (Tib. nyams), it is also recognized that "energies" can become disrupted through various types of meditation practice to such an extent that practitioners are given a Tibetan medical diagnosis of an imbalance or disorder of "rlung". Practitioners who associated ELSEs with a rlung imbalance typically reported other co-occurring somatic, affective, and cognitive phenomenology recognized by Tibetan medical nosology (Deane 2019; Jacobson 2007; Lindahl 2017). In the VCE study, appraisals of a rlung imbalance or rlung disorder were almost invariably tied to specific remedies intended to intervene upon dysregulated ELSEs, which will be explained below.

\subsubsection{Traditional Chinese Medicine}

Concepts associated with traditional Chinese medicine, most notably qi, were sometimes used by practitioners to appraise their experiences. $Q i$ was often specifically linked to the interpretive frameworks of traditional Chinese medicine, especially acupuncture, or to self-cultivation techniques, such as taiji and qigong. Meditation practitioners typically encountered these ideas when they were actively pursuing acupuncture as a therapeutic modality for mitigating or managing ELSEs, and, in some instances, this encounter significantly informed the overall interpretive framework they adopted. However, unlike Tibetan medical appraisals, the adoption of Chinese medical frameworks for the explanation or treatment of ELSEs occurred in roughly equal number across all Buddhist traditions, perhaps on account of its more widespread availability in the marketplace of alternative medicine. 


\subsubsection{Psychology and Neuroscience}

The psychological frameworks identified ELSEs with a psychological process, including emotional, relational, and sometimes trauma-specific themes. Some ELSEs were depicted in terms of "tension" or "blockage", followed by a "release", and such releases were sometimes characterized in socioemotional terms associated with the healing of relationships, distress, and old psychological wounds. Related to psychological interpretations, some practitioners employed neuroscientific explanations that referred to conceptions of brain-body interactions, neurological systems, the nervous system, and related constructs. One practitioner of vipassanā as taught by S. N. Goenka, who also had an educational background in cognitive science, described his ELSEs in the following way: "I mean, I'm in an extreme ecstasy state definitely for 45 minutes, and even then I just took a break from meditation because it was too much. The whole nervous system was like vibrating or the whole nervous system was active somehow". Despite the normative frameworks around "energies" and "winds" (Tib. rlung) available in Tibetan Buddhism, two practitioners in this tradition instead described their ELSEs in terms of a "nervous system imbalance" and as an "activation of my sympathetic nervous system".

\subsection{The Influence of Trajectories on Appraisals}

In some instances, meditation practitioners and meditation teachers alike thought it was important to determine whether ELSEs were normative, within the scope of expected meditation experiences, or resolvable through meditation practice, on the one hand, or potentially problematic and needing additional intervention, on the other (Lindahl et al. 2020). Appraisals and trajectories were linked in a variety of ways. For some practitioners, normative appraisals were sometimes maintained, even when ELSEs endured for a long duration, or were distressing, impaired functioning, or required intervention. However, for others, normative appraisals sometimes became difficult to maintain in the face of severe destabilization on account of ELSEs leading to co-occurring phenomenology, such as involuntary movements, a loss of sense of agency, insomnia, mania, and/or psychosis. For example, one Theravāda Buddhist practitioner reported first having ELSEs while on a long-term retreat, which then "went on for about five to six years until [they] finally settled down". Despite being familiar with the kundalini framework, in which "the narrative about this is that the energy is the kundalini that's coming up, and it's breaking through these blockages", he concluded, "I don't take much stock in that narrative". This is perhaps due to the fact that, for this practitioner, ELSEs were also a significant feature of another serious meditation-related challenge, which led first to elaborate "hallucinations" and delusions involving "an exaggerated sense of my own importance", then to disruptive "anti-social" behaviors, and, finally, to his removal from the retreat and hospitalization, at which point he was treated with antipsychotic medication. Though he initially understood his experiences with "energy" in Buddhist terms, as "getting rid of the defilements", his ultimate interpretation of his very challenging experiences was "I essentially went mildly insane".

In other instances, interpretations of ELSEs congruent with those put forth by religious traditions were held in tandem with frameworks from psychology, psychopathology, or neuroscience. Although one Theravāda Buddhist practitioner also described her experience in terms of kundalini, ultimately her view on ELSEs was: "I don't think they are a sign of enlightenment; I think they are energized neuronal states that happen to some people and don't happen to other people". Multiple practitioners stated that they believed that ELSEs could be understood as a process of purification or self-transformation and also could be associated with the onset of a manic or psychotic episode. For instance, one Tibetan Buddhist practitioner described how he thought "kundalini [ ... ] energy" was "dangerous stuff to play with", particularly because

once you awaken that, or if you intentionally go after it, you have no control over it! And it just sort of does what it wants to do. And it can really be incredibly 
painful. Incredibly disruptive. You know, it can absolutely cause psychosis. It just does a number on you.

Thus, normative appraisals could be articulated alongside approaches that acknowledge a need for intervention or an associated psychopathology.

\section{Remedies and Responses}

\subsection{Determining Need for Intervention}

Many practitioners reported that the nature of ELSEs or their impacts required determining whether there was need for intervention, an observation that also extends to the vast majority of meditation-related challenges documented in the VCE project (Lindahl et al. 2020). Although many meditation practitioners continued to hold normative appraisals of subtle body purification or spiritual emergency along the course of their challenging ELSEs, there were instances in which they decided that some kind of intervention outside of meditation practice was nevertheless warranted in order to make their experience more manageable. This was typically on account of severe or prolonged distress, functional impairment, an extensive duration, or a loss of control or inability to manage the experience on their own.

\subsection{Specific Remedies}

Mild and transient ELSEs were often described without mention of specific remedies, but for those experiences that were enduring, distressing, disruptive, or impairing of functioning, practitioners reported engaging with a wide range of strategies for managing ELSEs and their co-occurring phenomenology. Ways of working with ELSEs included drawing upon social relationships, changes in the type of meditation practice or the approach to meditation practice, various forms of embodied behaviors and therapies, grounding activities, dietary practices, and the use of medications.

\subsubsection{Acquiring an Interpretive Framework}

Often the first important step in coming to terms with ELSEs involved a meditation practitioner finding another person who could provide support, expertise, or simply empathy. Being given an interpretive framework alone could, in some instances, serve as a kind of remedy through alleviating confusion and associated distress. One Zen practitioner found that her main meditation teacher "didn't know what was going on" with her or "how to respond" to her ELSEs. By contrast, she described that "it was really helpful when [other expert's name] said, "This is kundalini'." She felt fortunate that her experience arose in a context where "I could get the guidance and help I needed". One Theravāda teacher thought it was very important to "normalize" ELSEs. Although she admitted that "it's not a usual thing", she felt it was important "not to pathologize it. It's not an illness. It isn't that they meditated wrong". Teachers were not the only source of normalization. Sometimes other practitioners could help someone to see that ELSEs were not "totally bizarre- that some of this stuff was almost expected side effects of meditating". Learning this could make the experience "less scary". For some practitioners, normalizing and removing the potential fear associated with ELSEs was "the most helpful thing". One practitioner of vipassanā in the tradition of S.N. Goenka described how receiving the "standard response" in her meditation tradition to "just observe" any unusual sensations that arise during practice was insufficient: "Not being able to put it in a suitable framework or context can be very distressing". Another practitioner in a Tibetan Buddhist tradition described his teacher as being "kind of resistant" to talking about kundalini. "He's kind of dismissive of it", which meant that "he wasn't entirely helpful about it", and conversations with this teacher "didn't go particularly well". This practitioner, and many others, had to find other resources in order to determine how to interpret and respond to what was happening. Having a context for understanding his ELSEs was described as helpful because "I don't think if I'd hadn't had that context, I would have been able to tolerate it". 


\subsubsection{Normalizing and Accepting}

Meditators also described various ways of responding to ELSEs within the context of meditation practice. Some believed that, like other meditative experiences, ELSEs are a "normal part of the process [ ... ] that's not good or bad. Or, the only time it becomes good or bad is if you either get really afraid of it or you get really caught up in it". Some were instructed to adopt an attitude to "just observe" the experience and to appraise it as "just sensation". Others with more enduring experiences found that surrendering to ELSEs was important. One even held the view that "ultimately kundalini becomes the guru". However, other meditators found that this way of responding to ELSEs was not helpful. One said she was "blinded" by the view that "'pleasant and unpleasant are going to come up, so just ignore them'-well, actually, not really, not necessarily".

\subsubsection{Practice-Related Remedies}

Meditators also attempted to navigate ELSEs through changes to their practice type or practice approach. One woman found that ELSEs went away when she changed her object of concentration. She also described that when "the energy would feel like it was building up", chanting practices would allow it to "release". Some found that standing or movement practices were also successful in "helping the energy move freely". In some cases, it was more apparent which practices were unhelpful, with one meditator reporting that, for him, visualization practices "would definitely make it worse", and that simple "formless" practices would "mostly still be okay". As noted above, related to this are numerous comments made on the association between ELSEs and the degree of effort applied in meditation practice. One Theravāda Buddhist practitioner thought "having a less tense brain" was important, as she needed to "not pounce on the breath", or her various ELSE-related problems would intensify. Similarly, another practitioner concluded that "the way I would practice [ ... ] actually should be more gentle". Others described needing to "back off", which in some cases meant adopting a gentler approach, but, in other cases, resulted in stopping practice altogether. One reported that he "took a break from meditation because it was too much". As has been demonstrated above, in a few more severe instances, reports of problematic ELSEs or their co-occurring phenomenology, such as involuntary movements or manic-seeming behaviors, led to practitioners being asked to leave a retreat on account of concerns that continuing meditation would exacerbate the problem.

\subsubsection{Acupuncture and Body Work}

Those for whom ELSEs endured beyond their meditation session sought out various ways of navigating, managing, or mitigating their effects. Some of these techniques come from systems that work specifically with the body or with subtle body energies. Multiple meditators described acupuncture as "very helpful". One practitioner, who reported having ongoing ELSE-related challenges for the last twelve years, stated that she receives acupuncture once a week because she "can't function without it". Others found similarly helpful results from other forms of body work, such as massage. Not surprisingly, many meditators turned to other practices that are intended to work directly with energy, such as qigong, taiji, or yoga, with mixed results. For some, these techniques helped them in their process of "straightening out energetically", as they learned to "circulate the energy more freely", or to "keep it flowing and keep it balanced". For one Tibetan Buddhist practitioner, who viewed his cluster of symptoms as a "rlung disorder", doing energy-related exercises is now a requirement to avoid physical pain and discomfort. However, others found that qigong practices would exacerbate energetic imbalances. One woman reported that they left her with "this terrible overstuffed feeling", and she reported being "very flummoxed" that she did not find these techniques to be helpful. 


\subsubsection{Trauma-Related Modalities}

Two practitioners found Somatic Experiencing, an embodied psychotherapeutic approach to resolving nervous system dysregulation, to be either "very helpful" or "tremendously helpful". According to the Somatic Experiencing model (Levine 1997; Payne et al. 2015), ELSEs are uncompleted sympathetic fight-or-flight action tendencies that were thwarted, resulting in a freeze response instead. Relaxation, meditation, immobility, and other states that simulate a freeze state have the potential to re-trigger sympathetic arousal, which could be experienced as ELSEs, involuntary movements, and other trauma-related phenomena. One practitioner, who for many years had been experiencing ELSEs accompanied by sleep changes and prolonged terror, described how, through the "slow-motion re-enacting of some of the trauma energies that were coming up", she was able "to begin to reconnect with here and now", which "helped me to begin to reintegrate back into a body".

\subsubsection{Grounding Activities}

A related cluster of remedies for ELSEs were characterized by their "grounding" effects. These included a range of dietary, physical, and even attentional interventions. Multiple practitioners described feeling "grounded" through establishing a physical connection with the earth, a recommendation that was also promoted by teachers in the study as a means to "allow the energy to be tapped off". Other practitioners undertook vigorous exercise or sequences of postural yoga oriented towards "cooling" the heat associated with ELSEs. One teacher recounted working with a meditator whose "body was vibrating". She drew upon her knowledge of bioenergetics therapy to orient the meditator into a bent-over posture that allowed the flow of "energy" to start "pulling down" toward her feet, because, in the estimation of this teacher, the meditator "didn't know how to ground it". Some practitioners also described grounding effects resulting from bathing or from being in large bodies of salt water.

\subsubsection{Dietary Changes}

Another grounding activity, and among the remedies more commonly prescribed by teachers and undertaken by practitioners, was dietary changes. A vegetarian diet, sugar, and caffeine were identified as exacerbating ELSEs and their co-occurring phenomenology, and a common antidote was for practitioners to introduce meat into their diet. One described how "it was quite amazing" because, within seconds of eating meat, he "actually did feel a lot better". Others ate more root vegetables to "pull the energy down", and some had to do "drastic things even just to have my digestive system work", including boiling flax into tea. In Tibetan Buddhist contexts, in which diagnoses of meditation-induced rlung disorder were made, practitioners were told that both meat and alcohol would help to rebalance them. One practitioner in Goenka's tradition of vipassanā ended up following the advice of the Tibetan Buddhist nun who appraised her experience as rlung imbalance: "I went straight out, got a big steak, bottle of red wine. I had a nice little party for one". Over a period of time, this led to a diminishment of her symptoms. Another Tibetan Buddhist practitioner described how his approach was to bring a "good dark beer" with him on retreat because "a beer, one time, really helps you bring down the energy. Believe it or not, it works".

\subsubsection{Medicines and Medications}

Practitioners also employed a range of medicines, both herbal medicines from healers practicing traditional Tibetan or Chinese forms of medicine, as well as modern pharmaceuticals. For some, herbal medicines "seemed to help somewhat", whereas for others they "helped a lot". Multiple meditators, especially in the context of retreat, expressed a need to take sleeping pills when ELSEs became so prolonged and intense that they affected the ability to sleep. As discussed above in the section on impacts, insomnia could also lead to further destabilization, and some of these same practitioners were ultimately also given antipsychotic medications, such as Zyprexa, or antianxiety medications, such as Ativan, 
after they were removed from retreats. Other practitioners mentioned that Klonopin, another antianxiety medication, was helpful in addressing energy symptoms. However, one of them summed up the stigma associated with using medications by explaining: "I've used medications, and I think those are helpful. And in the meditation community that's a lot of times shunned and looked down upon, and you're a failure if you actually have to use Klonopin".

\section{Conclusions}

Although there are historical and textual references to ELSEs in Buddhist traditions, and even a few psychological studies of related phenomena, this paper, based upon a large qualitative study of meditation-related challenges, offers a unique glimpse into ELSEs in the lives of contemporary meditation practitioners and how they are regarded by meditation teachers.

Among practitioners of Buddhist meditation reporting challenging experiences in the VCE study, ELSEs are a relatively common phenomenology, with $62 \%(42 / 68)$ of practitioners spontaneously reporting ELSEs at least once. Further research is needed to determine the frequency of ELSEs in other samples, such as novice meditators versus advanced practitioners, or across different meditation traditions. The descriptions of ELSEs were often presented in emic terms, such as kundalini, prāna, rlung, or, qi, but practitioners also invoked a wide range of metaphors in an attempt to describe these unusual experiences. ELSEs had various impacts and trajectories for practitioners that depended on a number of factors, the most common of which were the type and amount of meditation practice. For some, ELSEs were transient or were mentioned only in passing, whereas, for others, they were enduring and central to their main narrative of meditation-related challenges. ELSEs co-occurred with other salient changes, especially in the somatic, affective, cognitive, and perceptual domains. ELSEs could be positive and enhancing or distressing and impairing. Some practitioners thought that ELSEs were congruent with normative models found within, and beyond, Buddhist traditions, such as perceiving subtle sensations, working with the subtle body energies, and related purification frameworks. Other ELSEs were described using emic conceptions of normal-but-not-normative experiences, such as nyams and meditation sickness. However, Buddhist traditions do not have the same emic models for interpreting and managing ELSEs, nor do these models appear to be equally available among meditation practitioners in the West. This led many practitioners to seek interpretive frameworks and suitable responses from outside their tradition. Although a small body of medical and psychiatric literature has assessed for, and documented, ELSEs, these remain little known anomalous experiences, and biomedical appraisals for ELSEs were generally not made available to the practitioners who sought out, or were involuntarily placed in, medical or psychiatric care. Practitioners, thus, also came to entertain spiritual emergency frameworks as a way of making sense of their experience. Especially in instances in which a suitable intervention was sought, some practitioners adopted appraisals from the domains of traditional medicine, whether a Tibetan rlung disorder diagnosis, or notions of $q i$ associated with acupuncture in traditional Chinese medicine. Occasionally, frameworks from Western psychology, psychiatry, and even neuroscience were preferred, or were articulated, alongside other appraisals from Buddhist or spiritual emergency frameworks. Congruent with attribution-based approaches to the study of experiences deemed religious (Taves 2009), this study of ELSEs suggests that such phenomena are not universally appraised as religious; instead, ascriptions of religious significance and value were made in certain contexts and circumstances, but not in others.

In light of the heterogeneous phenomenology of ELSEs, their range of impacts, and the various appraisals and responses associated with them, researchers (as well as meditation teachers) should perhaps resist the temptation to assume a spiritual emergency "kundalini awakening" framework and the positive spiritual trajectory it typically implies. The archetypal notion popularized by Grof and Grof ([1986] 2017), and developed through subsequent research in transpersonal psychology in particular, seems to capture neither 
the full range of ELSEs nor their various trajectories. Rather, as we have argued elsewhere (Lindahl et al. 2021), it may be more appropriate to adopt a person-centered approach that takes the worldviews, values, and proximate and ultimate goals of the practitioner into account when considering which appraisals and remedies to offer. Furthermore, as documented elsewhere in the VCE study (Lindahl et al. 2020), determining the best response or intervention for managing ELSEs was, in many cases, more salient than determining the correct appraisal. The interpretations that practitioners gave to ELSEs were often dictated by remedies or responses that worked for managing them. Helpful remedies included normalizing ELSEs, changes in practice type or approach, acupuncture, herbal and pharmacological medicines, and various grounding activities, such as exercise and a change in diet.

Given that cautions about the risks of engaging in subtle body or energetic practices are well known, at least within Vajrayāna Buddhist traditions, and that other Buddhist traditions also have ways of normalizing some ELSEs, it is perhaps not surprising that, in some cases, practitioners maintained a normative appraisal of ELSEs, even in the face of significant disruption to their lives and to their meditation practice. Nevertheless, some practitioners reported having to manage ELSEs over the course of many years or in an ongoing way, regardless of whether they thought the experiences were normative and desirable, a nuisance, or ultimately unpleasant and undesirable. Those practitioners who reported severely impairing and negatively impactful ELSEs often had difficulty understanding them as normative in the terms presented within Buddhism (e.g., part of purification), or beyond Buddhism (e.g., as a kundalini awakening) (see also Lindahl (2017)). Instead, there were instances in which practitioners looked to modern psychology, neuroscience, and psychopathology instead of, or in addition to, either emic Buddhist frameworks, or the spiritual emergency frameworks that have more recently been employed to understand such anomalous experiences. However, the availability of Buddhist models for understanding and managing ELSEs does not imply that they are not potentially of clinical significance. Indeed, in some cases, ELSEs co-occurred with additional challenging phenomenology, such as involuntary movements, mania, and delusions, and, as with other meditation-related challenges documented in this study, the time course, severity, and degree of distress or functional impairment were also identified as factors influencing whether ELSEs needed additional support or intervention beyond what a Buddhist teacher or tradition could offer (Lindahl et al. 2020).

It is worth noting that the frequency of ELSEs was roughly equivalent across practitioners from different Buddhist traditions, and yet normative frameworks for understanding and working with them differed significantly depending upon the Buddhist lineage. This may indicate that ELSEs may occur regardless of the expectation effects from specific practices or associated worldviews. Further research is needed to establish a possible mechanism by which various meditation practices could lead to ELSEs, as well as whether different practices lead to different types of ELSEs.

If ELSEs are relatively common challenges that are not differentially associated with specific approaches to meditation, or with specific worldviews or expectations, then one might expect different Buddhist lineages to have acknowledged them as an effect of meditation and to have developed means of working with them. The limited data from the VCE study on Western Buddhist meditators and meditation teachers cannot account for why certain practitioners-specifically those in Zen and Theravāda lineages outside of that of S.N. Goenka-reported less engagement with emic models for appraising or managing ELSEs and tended to look elsewhere. However, research on Buddhist meditation traditions and Buddhist modernism may offer possible explanations for why ELSEs do not seem to be commonly recognized, or ascribed significance, in certain Buddhist lineages, as currently constituted in the West. Given the particular epistemic space of modern Buddhism in the West, which bridges a range of epistemologies, both religious and spiritual, as well as psychological and scientific (McMahan 2008; Wilson 2014), it is unsurprising that meditation practitioners who were not given emic frameworks looked beyond Buddhist 
traditions in order to make sense of unusual, unexpected, and challenging ELSEs. However, the reason why these frameworks were unavailable to certain practitioners in the first place may have more to do with the current status of the transmission of Buddhism from Asia to the West, both in terms of the availability of textual sources and meditation instruction. As Greene (2021) has pointed out, Western perceptions of Buddhism and Buddhist meditation by scholars and practitioners have been influenced by the specific texts that have been identified as "canonical representations of traditional Buddhist meditation literature". To date, this has not included sufficient representation of texts on meditation-related challenges and how to work with them. Although some depictions of meditation sickness involving ELSEs in Zen lineages have become more "canonical", such as Hakuin (2009), nevertheless, most meditation-related challenges remain beyond the general conception of the anticipated effects among meditation practitioners in the West. Greene's translation of a text on remedies for meditation sickness, for example, begins to address this lacuna. Considering the few references to meditation sickness among Zen practitioners and teachers in the VCE study, the various strategies for working with meditation sickness that can be found in textual sources, such as Greene (2021), may also have been downplayed, if not lost, among contemporary lineages. This might also account for the unequal representation of frameworks for ELSEs across the Buddhist lineages represented in the VCE study, despite the relatively consistent frequency of their occurrence. Additional translations of Asian language textual sources, as well as additional social scientific research among both Western and Asian Buddhist communities, are needed to better understand the frequency of, interpretations of, and responses to meditation-related challenges, including those that could be characterized as energy-like somatic experiences.

Author Contributions: Conceptualization, W.B.B.; Data curation, D.J.C., J.R.L. and W.B.B.; Formal analysis, D.J.C., J.R.L., R.P. and W.B.B.; Funding acquisition, W.B.B. and J.R.L.; Investigation, W.B.B. and J.R.L.; Methodology, W.B.B. and J.R.L.; Writing-original draft, D.J.C., J.R.L., R.P. and W.B.B.; Writing-review \& editing, D.J.C., J.R.L., R.P. and W.B.B. All authors have read and agreed to the published version of the manuscript.

Funding: This analysis was not supported by external funding.

Institutional Review Board Statement: The study was conducted according to the guidelines of the Declaration of Helsinki, and approved by the Institutional Review Board (or Ethics Committee) of Brown University (protocol code 1301000752, approved 15 August 2016).

Informed Consent Statement: Informed consent was obtained from all subjects involved in the study.

Data Availability Statement: Data sharing is not applicable to this article.

Conflicts of Interest: W.B.B. is a MBSR and MBCT teacher and has received financial compensation for this role. W.B.B. is nominally affiliated with the Mindfulness Center at Brown University which generates income by offering mindfulness classes to the public. W.B.B. is the founder of Cheetah House, a RI non-profit organization that provides information about meditation-related challenges, individual consultations, and support groups, as well as educational trainings to meditation teachers, clinicians, educators and mindfulness providers. J.R.L.'s spouse is the founder of Cheetah House. J.R.L. has also received compensation for providing educational trainings on meditation-related challenges. This interest has been disclosed to and is being managed by Brown University, in accordance with its Conflict of Interest and Conflict of Commitment policies. The other authors declare no conflict of interest.

\section{Notes}

1 Also included in the present analysis are an additional eight practitioners and one expert from a replication study of meditationrelated challenges in the vipassanā tradition of S.N. Goenka.

2 For detailed inclusion and exclusion criteria, see Lindahl et al. (2017), Supplemental File S4: Phenomenology codebook. 
3 It is worth noting that, because the interviews were exploratory and semi-structured, they did not query each phenomenology category directly. Therefore, it is possible that other practitioners also had experiences that would have met criteria for "somatic energy", but did not include descriptions of them in their main narrative about meditation-related challenges.

\section{References}

Ahn, Juhn Y. 2021. Meditation Sickness. In The Oxford Handbook of Meditation. Edited by Miguel Farias, David Brazier and Mansur Lalljee. Oxford: Oxford University Press, pp. 887-906.

Albanese, Catherine L. 2007. A Republic of Mind and Spirit: A Cultural History of American Metaphysical Religion. New Haven: Yale University Press.

Amihai, Ido, and Maria Kozhevnikov. 2014. Arousal vs. Relaxation: A Comparison of the Neurophysiological and Cognitive Correlates of Vajrayana and Theravada Meditative Practices. PLoS ONE 9: e102990. [CrossRef]

Benedict, Adriana Lee, Linda Mancini, and Michael A. Grodin. 2009. Struggling to Meditate: Contextualising Integrated Treatment of Traumatised Tibetan Refugee Monks. Mental Health, Religion \& Culture 12: 485-99.

Brefczynski-Lewis, Julie A., Antoine Lutz, Hillary S. Schaefer, Daniel B. Levinson, and Richard J. Davidson. 2007. Neural Correlates of Attentional Expertise in Long-Term Meditation Practitioners. Proceedings of the National Academy of Sciences 104: 11483-88. [CrossRef] [PubMed]

Buttner, Phillip Ross. 2016. A Precarious Path: An Inquiry into the Travails Faced by Westerners Practicing Theravadin Buddhism. Ph.D. thesis, Institute of Transpersonal Psychology, Palo Alto, CA, USA.

Cayton, Amy. 2007. Balanced Mind, Balanced Body: Anecdotes and Advice from Tibetan Buddhist Practitioners on Lung. Portland: Foundation for the Preservation of the Mahayana Tradition.

Chaoul, Marco Alejandro. 2006. Magical Movements ('Phrul 'Khor): Ancient Yogic Practices in the Bön Religion and Contemporary Medical Perspectives. Ph.D. thesis, Rice University, Houston, TX, USA.

Cozort, Daniel. 1986. Highest Yoga Tantra: An Introduction to the Esoteric Buddhism of Tibet. Ithaca: Snow Lion Publications.

Daniel, E. Valentine. 1994. The Individual in Terror. In Embodiment and Experience: The Existential Ground of Culture and Self. Edited by Thomas J. Csordas. Cambridge: Cambridge University Press, pp. 229-47.

Deane, Susannah. 2019. rLung, Mind, and Mental Health: The Notion of "Wind" in Tibetan Conceptions of Mind and Mental Illness. Journal of Religion and Health 58: 708-24. [CrossRef] [PubMed]

Dor-Ziderman, Yair, Aviva Berkovich-Ohana, Joseph Glicksohn, and Abraham Goldstein. 2013. Mindfulness-Induced Selflessness: A MEG Neurophenomenological Study. Frontiers in Human Neuroscience 7: 582. [CrossRef] [PubMed]

Gerke, Barbara. 2013. On the 'Subtle Body' and 'Circulation' in Tibetan Medicine. In Religion and the Subtle Body in Asia and the West: Between Mind and Body. Edited by Geoffrey Samuel and Jay Johnston. London: Routledge, pp. 83-99.

Goenka, S. N. 2000. The Discourse Summaries. Onalaska: Vipassana Research Publications. First published 1987.

Goretzki, Monika, Michael A. Thalbourne, and Lance Storm. 2013. Development of a Spiritual Emergency Scale. Journal of Transpersonal Psychology 45: 105-17.

Greene, Eric M. 2021. The Secrets of Buddhist Meditation: Visionary Meditation Texts from Early Medieval China. Honolulu: University of Hawaii Press.

Greyson, Bruce. 2000. Some Neurophysiological Correlates of the Physio-Kundalini Syndrome. Journal of Transpersonal Psychology 32: 123-34.

Grof, Christina, and Stanislav Grof. 2017. Spiritual Emergency: The Understanding and Treatment of Transpersonal Crises. International Journal of Transpersonal Studies 36: 30-43. First published 1986. [CrossRef]

Gross, Gisela, Gerd Huber, Joachim Klosterkötter, and Maria Linz. 2008. BSABS: Bonn Scale for the Assessment of Basic Symptoms. Aachen: Shaker Verlag.

Gyatso, Janet. 2015. Being Human in a Buddhist World: An Intellectual History of Medicine in Early Modern Tibet. New York: Columbia University Press.

Hakuin. 2009. Idle Talk on a Night Boat. In Hakuin's Precious Mirror Cave: A Zen Miscellany. Edited by Norman Waddell. Berkeley: Counterpoint.

Hanegraaff, Wouter J. 1996. New Age Religion and Western Culture: Esotericism in the Mirror of Secular Thought. Studies in the History of Religions. Leiden and New York: E.J. Brill.

Hydén, Lars Christer. 1995. The Rhetoric of Recovery and Change. Culture, Medicine, and Psychiatry 19: 73-90. [CrossRef]

Jacobson, Eric. 2007. 'Life-Wind Illness' in Tibetan Medicine: Depression, Generalized Anxiety, and Panic Attack. In Soundings in Tibetan Medicine: Anthropological and Historical Perspectives, Proceedings of the Tenth Seminar of the International Association for Tibetan Studies. Edited by Mona Schrempf. Leiden: Brill, pp. 223-43.

Jung, Carl Gustav. 1996. The Psychology of Kundalini Yoga: Notes of the Seminar Given in 1932 by C.G. Jung. Bollingen Series; Princeton: Princeton University Press.

Kaselionyte, Justina, and Andrew Gumley. 2017. "It's Like a Charge-Either Fuses You or Burns You Out": An Interpretative Phenomenological Analysis of Extreme Mental States in Meditation Context. Mental Health, Religion E Culture 20: 986-1001.

Kongtrul, Jamgön. 2007. The Treasury of Knowledge: Book Eight, Part Four: Esoteric Instructions: A Detailed Presentation of the Process of Meditation in Vajrayāna. Translated by Sarah Harding. Ithaca: Snow Lion Publications. 
Kozhevnikov, Maria, James Elliott, Jennifer Shephard, and Klaus Gramann. 2013. Neurocognitive and Somatic Components of Temperature Increases During gTummo Meditation: Legend and Reality. PLoS ONE 8: e58244. [CrossRef]

Krishna, Gopi. 1967. Kundalini: The Evolutionary Energy in Man. New Delhi: Ramadhar \& Hopman.

Landau, Mark J. 2018. Using Metaphor to Find Meaning in Life. Review of General Psychology 22: 1. [CrossRef] [PubMed]

Levine, Peter. 1997. Waking the Tiger: Healing Trauma: The Innate Capacity to Transform Overwhelming Experiences. Berkeley: North Atlantic Books.

Lindahl, Jared R. 2017. Somatic Energies and Emotional Traumas: A Qualitative Study of Practice-Related Challenges Reported by Vajrayāna Buddhists. Religions 8: 153. [CrossRef]

Lindahl, Jared R., Nathan E. Fisher, David J. Cooper, Rochelle K. Rosen, and Willoughby B. Britton. 2017. The Varieties of Contemplative Experience: A Mixed-Methods Study of Meditation-Related Challenges in Western Buddhists. PLoS ONE 12: e0176239. [CrossRef] [PubMed]

Lindahl, Jared R., David J. Cooper, Nathan E. Fisher, Laurence J. Kirmayer, and Willoughby B. Britton. 2020. Progress or Pathology? Differential Diagnosis and Intervention Criteria for Meditation-Related Challenges: Perspectives from Buddhist Meditation Teachers and Practitioners. Frontiers in Psychology 11: 1905. [CrossRef]

Lindahl, Jared R., Willoughby B. Britton, David J. Cooper, and Laurence J. Kirmayer. 2021. Challenging and Adverse Meditation Experiences: Toward a Person-Centered Approach. In The Oxford Handbook of Meditation. Edited by Miguel Farias, David Brazier and Mansur Lalljee. Oxford: Oxford University Press, pp. 841-64.

Lingpa, Düdjom. 2015. The Vajra Essence: Düdjom Lingpa's Visions of the Great Perfection. Translated by B. Alan Wallace. Boston: Wisdom Publications.

Llewellyn-Beardsley, Joy, Stefan Rennick-Egglestone, Felicity Callard, Paul Crawford, Marianne Farkas, Ada Hui, David Manley, Rose McGranahan, Kristian Pollock, Amy Ramsay, and et al. 2019. Characteristics of Mental Health Recovery Narratives: Systematic Review and Narrative Synthesis. PLoS ONE 14: e0214678. [CrossRef]

Lopez, Donald S. 2008. Buddhism and Science: A Guide for the Perplexed. Chicago: University of Chicago Press.

Lutz, Antoine, Julie Brefczynski-Lewis, Tom Johnstone, and Richard J. Davidson. 2008. Regulation of the Neural Circuitry of Emotion by Compassion Meditation: Effects of Meditative Expertise. PLoS ONE 3: e1897. [CrossRef]

MacLean, Katherine A., Emilio Ferrer, Stephen R. Aichele, David A. Bridwell, Anthony P. Zanesco, Tonya L. Jacobs, Brandon G. King, Erika L. Rosenberg, Baljinder K. Sahdra, Phillip R. Shaver, and et al. 2010. Intensive Meditation Training Improves Perceptual Discrimination and Sustained Attention. Psychological Science 21: 829-39. [CrossRef]

McAdams, Dan P., Jeffrey Reynolds, Martha Lewis, Allison H. Patten, and Phillip J. Bowman. 2001. When Bad Things Turn Good and Good Things Turn Bad: Sequences of Redemption and Contamination in Life Narrative and Their Relation to Psychosocial Adaptation in Midlife Adults and in Students. Personality and Social Psychology Bulletin 27: 474-85. [CrossRef]

McMahan, David L. 2008. The Making of Buddhist Modernism. Oxford: Oxford University Press.

McMahan, David L. 2011. Buddhism as the "Religion of Science": From Colonial Ceylon to the Laboratories of Harvard. In Handbook of Religion and the Authority of Science. Edited by James R. Lewis and Olav Hammer. Leiden: Brill, pp. 115-40.

Musolff, Andreas. 2017. Metaphor and Cultural Cognition. In Advances in Cultural Linguistics. Edited by Farzad Sharifian. Singapore: Springer, pp. 325-44.

Nyboe, Lene, Marianne K. Moeller, Claus H. Vestergaard, Hans Lund, and Poul Videbech. 2016. Physical Activity and Anomalous Bodily Experiences in Patients with First-Episode Schizophrenia. Nordic Journal of Psychiatry 70: 514-20. [CrossRef]

Ondish, Peter, Dov Cohen, Kay Wallheimer Lucas, and Joseph Vandello. 2019. The Resonance of Metaphor: Evidence for Latino Preferences for Metaphor and Analogy. Personality and Social Psychology Bulletin 45: 1531-48. [CrossRef]

Ossoff, Jon. 1993. Reflections of Shatkipat: Psychosis or the Rise of Kundalini? Journal of Transpersonal Psychology 25: $29-42$.

Palitsky, Roman, Daniel Sullivan, Isaac F. Young, and Sheila Dong. 2019. Worldviews and the Construal of Suffering from Depression. Journal of Theoretical Social Psychology 3: 191-208. [CrossRef]

Parnas, Josef, Paul Møller, Tilo Kircher, Jørgen Thalbitzer, Lennart Jansson, Peter Handest, and Dan Zahavi. 2005. EASE: Examination of Anomalous Self-Experience. Psychopathology 38: 236-58. [CrossRef]

Payne, Peter, Peter A. Levine, and Mardi A. Crane-Godreau. 2015. Somatic Experiencing: Using Interoception and Proprioception as Core Elements of Trauma Therapy. Frontiers in Psychology 6: 93. [PubMed]

Rinpoche, Tsoknyi. 2012. Open Heart, Open Mind. New York: Random House.

Samuel, Geoffrey. 2008. The Origins of Yoga and Tantra: Indic Religions to the Thirteenth Century. Cambridge and New York: Cambridge University Press.

Samuel, Geoffrey. 2019. Unbalanced Flows in the Subtle Body: Tibetan Understandings of Psychiatric Illness and How to Deal with It. Journal of Religion and Health 58: 770-94. [CrossRef]

Samuel, Geoffrey, and Jay Johnston. 2013. Religion and the Subtle Body in Asia and the West: Between Mind and Body. Routledge Studies in Asian Religion and Philosophy; Abingdon and New York: Routledge.

Sanches, Laura, and Michael Daniels. 2008. Kundalini and Transpersonal Development: Development of a Kundalini Awakening Scale and a Comparison between Groups. Transpersonal Psychology Review 12: 73-83.

Sannella, Lee. 1987. The Kundalini Experience: Psychosis or Transcendence? Lower Lake: Integral Publishing. First published 1978.

Sayadaw, Mahasi. 2016. Manual of Insight. Translated by Vipassanā Mettā Foundation Translation Committee. Somerville: Wisdom Publications. 
Sferra, Francesco. 1999. The Concept of Purification in Some Texts of Late Indian Buddhism. Journal of Indian Philosophy 27: 83-103. [CrossRef]

Sharf, Robert H. 2015. Is Mindfulness Buddhist? (and Why It Matters). Transcultural Psychiatry 52: 470-84. [CrossRef] [PubMed]

Taves, Ann. 2009. Religious Experience Reconsidered: A Building-Block Approach to the Study of Religion and Other Special Things. Princeton: Princeton University Press.

Taylor, Steve. 2015. Energy and Awakening: A Psycho-Sexual Interpretation of Kundalini Awakening. Journal of Transpersonal Psychology 47: 219-41.

Valanciute, A., and L. Thampy. 2011. Physio Kundalini Syndrome and Mental Health. Mental Health, Religion E Culture 14: 839-42.

Waldron, William S. 2017. Reflections on Indian Buddhist Thought and the Scientific Study of Meditation, or: Why Scientists Should Talk More with Their Buddhist Subjects. In Meditation, Buddhism, and Science. Edited by David McMahan and Erik Braun. Oxford: Oxford University Press, pp. 84-113.

White, David Gordon. 1996. The Alchemical Body: Siddha Traditions in Medieval India. Chicago: University of Chicago Press.

Wilson, Jeff. 2014. Mindful America: The Mutual Transformation of Buddhist Meditation and American Culture. Oxford: Oxford University Press.

Woollacott, Marjorie H., Yvonne Kason, and Russell D. Park. 2020. Investigation of the Phenomenology, Physiology and Impact of Spiritually Transformative Experiences-Kundalini Awakening. Explore 17: 525-34. [CrossRef] [PubMed]

Zysk, Kenneth G. 1993. The Science of Respiration and the Doctrine of the Bodily Winds in Ancient India. Journal of the American Oriental Society 113: 198-213. [CrossRef] 